\title{
Experimental and numerical simulation of diesel engine cylinder liner with laser quenching
}

\author{
Shengwen ZHANG, Zifan XIA
}

\begin{abstract}
Using Abaqus to simulate the high-phosphorus cast iron laser quenching process, the distribution of quenching temperature field was analyzed. It was found that the temperature difference at the beginning of scanning was large. The control scanning speed was proposed to solve the uneven distribution of hardened layer, and the hardening depth was predicted. The simulation results were verified by laser quenching test. The results show that the test results are good when the scanning power is $1100 \mathrm{~W}$ and the scanning speed is $25 \mathrm{~mm} / \mathrm{s}$.
\end{abstract}

Index Terms - Moving heat source; laser quenching; moving heat source.

\section{INTRODUCTION}

Laser quenching is to irradiate the surface of the part with a high energy density laser beam, so that the hardened layer part absorbs the light energy instantaneously and immediately converts it into heat energy, so that the temperature of the laser action area rises sharply above the phase transition point of the material to form austenite [1]. The laser characterization of the sample after laser quenching and the use of computer technology to simulate the laser quenching process are currently two research trends in laser quenching technology. In the research part of the laser quenching technology based on the experiment, McDaniels et al. performed laser quenching on AISI4340 and analyzed the fatigue strength and microstructure after quenching. The results show that the heat affected zone of laser quenching technology does not work on high Cycle working materials. Fatigue strength causes adverse effects [2], After laser hardening of cast iron, Stanislav found that its surface hardness and wear resistance were significantly improved compared with conventional surface strengthening techniques, and described the phase transition near graphite. This description relates to the formation of hard and carbide phases, resulting in hardness values above $65 \mathrm{HRc}$ and good wear resistance [3]. In the numerical simulation of laser quenching, the rapid development of computer control technology has brought a lot of research results to the research of numerical simulation in laser quenching process, which provides a good theoretical basis for numerical simulation technology in laser quenching application $[4,5]$. Wang Zhou et al. of Shanghai Jiaotong University used Abaqus/Standard to carry out finite element simulation of laser quenching of AISI4101 steel. In the physical model, the effects of different factors such as different thermal boundary conditions of laser

Shengwen Zhang is a professor and master advisor of Jiangsu University of Science and Technology. He received his Ph.D. degree in mechanical manufacturing in Jiangsu University in 2013. The main research directions are CAD/CAPP/CAM integration technology and Group Technology.

Zifan Xia is a Master student of Jiangsu University of Science and Technology. The main research directions was Laser surface strengthening technology. heat source and workpiece surface were considered. A more reasonable laser quenching process to predict the hardened layer of the quenched sample [6], Hamar et al. proposed and solved the three-dimensional model of steel body laser hardening and its induction preheating. The model consists of two partial differential equations that describe the distribution of nonlinear and non-stationary magnetic fields in the system and the non-stationary temperature field in the heated object. The two equations are solved numerically in a hard-coupled formulation, respecting the temperature dependence of the material parameters and using typical examples [7], Duradundi Sawant Badkar et al. applied the response surface method and the central combination design method to model the effects of laser quenching parameters on the heat input and quenching layer, and optimized the optimal process parameters for laser quenching of titanium plates [8].

Select the diesel engine cylinder liner sample to simulate the finite element temperature field of the laser surface quenching process, establish a three-dimensional model, comprehensively consider various real environments, and use Abaqus' various post-processing functions to comprehensively analyze the simulation results, and then pass the test verification. The authenticity of the simulation results. The combination of simulation and experimentation makes the development of laser quenching technology based on a reliable and solid theoretical foundation.

\section{TEST MATERIALS AND METHODS}

The test cylinder liner is from a certain type of marine medium speed diesel engine. The test material in this study is the diesel engine block casting blank for the factory. The sample size is $39 \mathrm{~mm} * 23 \mathrm{~mm} * 10 \mathrm{~mm}$, and the sample equipment is IPG YLS-6000 fiber laser with a spot area of $5 \mathrm{~mm} * 5 \mathrm{~mm}$.

The sample was ultrasonically cleaned and dried by acetone solution before the laser quenching test. In the experiment, the constant laser scanning spot area is $5 \mathrm{~mm} \times 5 \mathrm{~mm}$, and the combination of different scanning power and scanning speed parameters is selected for laser quenching experiment. The laser scanning power was selected from $1100-1500 \mathrm{~W}$ with a gradient of $100 \mathrm{~W}$; the scanning speed was $25-45 \mathrm{~mm} / \mathrm{s}$ with a gradient of $5 \mathrm{~mm} / \mathrm{s}$. After the initial laser quenching test, the laser quenching test parameter combination of excessively high scanning power or too small scanning speed caused by melting of the quenching layer and intermittent phenomenon of thermal instability and hardening due to excessive scanning power or excessive scanning speed was excluded. The test parameters are selected according to the appearance of the laser quench hardening zone, and then the hardness of the quenched sample is measured and the microstructure is observed. According to the test results of the process, the samples with excellent appearance, surface hardness and metallographic analysis of the hardened zone 
were tested for friction and wear properties. Finally, the optimal test parameters for improving the wear resistance of the diesel engine liner by laser quenching are determined.

\section{TEST RESULTS AND ANALYSIS}

After the laser quenching, the hardness of the sample was tested, and the sample was cut perpendicular to the laser scanning path to cut the cross section of the water mill and polished to a metallographic microscope to observe no significant scratches. The hardness of the sample was measured with a MH-50 microhardness tester and the hardness of the measurement point was recorded. The microhardness of the quenched sample is measured from the 40um from the quenched surface layer. The hardness value is measured three times from the quenching layer to the material matrix every 40um. The average hardness of the point is taken as the hardness value of the measuring point. The measurement is terminated until three consecutive points appear without exceeding the matrix hardness range value.

The hardness measurement showed a good hardness gradient value at a scan power of $1100 \mathrm{~W}$ and a scan speed of $25 \mathrm{~mm} / \mathrm{s}$, as shown in Fig.1. The portion with microhardness greater than the matrix can be defined as the hardened region [9], and the reference matrix hardness is about $320 \mathrm{HV}$. The hardness gradient map shows that the quench hardened layer has a depth of about $0.4 \mathrm{~mm}$.

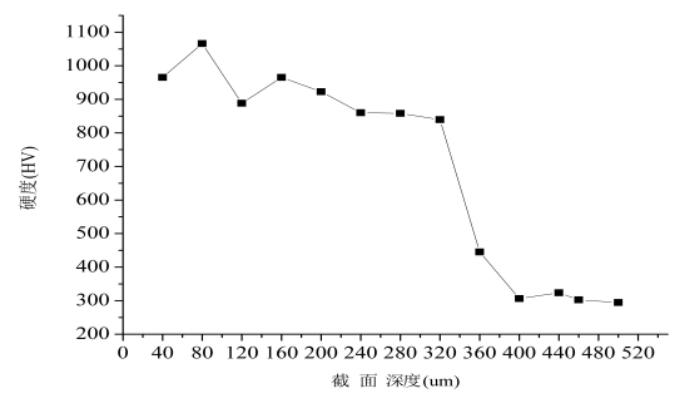

Fig.1. Section hardness distribution curve

\section{NUMERICAL SIMULATION AND ANALYSIS}

\subsection{Pretreatment}

The laser quenching temperature field is a typical nonlinear transient heat transfer problem, so the laser scanning temperature field follows the Fourier heat conduction differential equation:

$$
\frac{\partial}{\partial x}\left(\lambda \frac{\partial T}{\partial x}\right)+\frac{\partial}{\partial y}\left(\lambda \frac{\partial T}{\partial y}\right)+\frac{\partial}{\partial z}\left(\lambda \frac{\partial T}{\partial z}\right)+Q=\rho C_{p} \frac{\partial T}{\partial t}
$$

$\rho$ is the material density, $\boldsymbol{C}_{p}$ is the specific heat capacity of the material, $\lambda$ is the thermal conductivity of the material, and $Q$ is the latent heat of phase change.

The cylinder liner sample is a new type of wear-resistant cast iron developed by adding $\mathrm{P}$ element to the factory. The thermal properties of the cast iron have no data reference, so this paper uses Jmatpro software to calculate the thermal properties of high-phosphorus cast iron. The high-phosphorus cast iron material composition was input into the cast iron module function of the jmatpro software, and then the parameters of the density, thermal expansion coefficient, specific heat capacity and thermal conductivity of the high-phosphorus cast iron were imported into the Abaqus material property module.
Using JmatPro software to calculate the tissue change of the temperature rise process for high-phosphorus cast iron materials with a known composition ratio, The relationship between austenite conversion rate and temperature change of high phosphorus cast iron material is shown in Fig. 2. The critical temperature of the high phosphorus cast iron material is $\mathrm{A}_{\mathrm{C} 1}=732^{\circ} \mathrm{C}, \mathrm{A}_{\mathrm{C} 3}=797^{\circ} \mathrm{C}$. Referring to the quenching temperature requirement, the heating temperature should be guaranteed at around 30 to $50{ }^{\circ} \mathrm{C}$ above Ac3. Considering that the cast iron material is optimized and optimized by the factory, the melting point of the material cannot be obtained. The melting point of most cast iron is about $1400{ }^{\circ} \mathrm{C}$. Therefore, the critical point temperature of the quenching phase change of the high-phosphorus cast iron sample is about $850{ }^{\circ} \mathrm{C}$, and the melting point is about $1400{ }^{\circ} \mathrm{C}$. Thereby, the depth of the quench hardened layer is predicted by simulating the quenching temperature field.

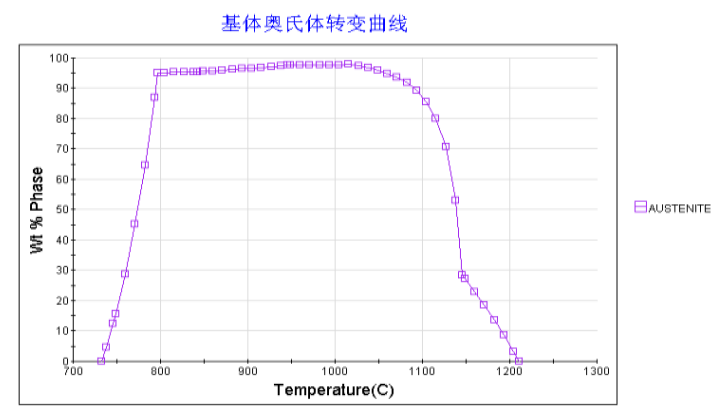

Fig.2. High-phosphorus cast iron austenite transformation curve

Before the moving heat source is loaded, set the initial temperature condition of the sample substrate to $25^{\circ} \mathrm{C}$, In the laser quenching process of the base sample, the laser spot is a rectangular spot with uniform energy distribution. Therefore, in the numerical simulation of this paper, the power density of the laser heat source is

$$
q=\frac{p}{A \times B}
$$

The laser radiation density of the base sample from the start position to the end position in the scanning direction of the spot:

$$
Q=\eta \times \frac{p}{A \times B} \times \frac{A}{v}
$$

Where $\mathrm{P}$ is the laser power, $\mathrm{V}$ is the spot scanning speed, and $\eta$ is the absorption rate of the material by the laser. The absorption rate of the material selected for the fiber laser is $0.35[10]$.

\subsection{Load application process}

The prototype size is $39 \mathrm{~mm}$ long, $23 \mathrm{~mm}$ wide and $10 \mathrm{~mm}$ high. In order to reduce the calculation time and data volume when modeling the sample in Abaqus, the sample is divided into two symmetrical halves by the central trajectory of the spot scanning path. After the creation, the model size is $39 \mathrm{~mm} * 11.5 \mathrm{~mm} * 10 \mathrm{~mm}$, the built model is given the material properties, and then the model is meshed. Considering the calculation accuracy and calculation efficiency of Abaqus analysis, the laser heated area is a fine mesh, and the heat affected zone and other areas are divided into coarse mesh from the inside to the outside, as shown in Fig. 3 below. 


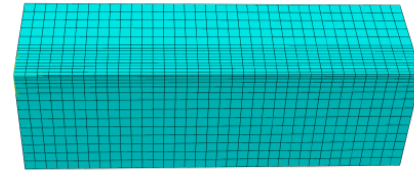

Fig.3. Model meshing diagram

Temperature field simulation According to the actual processing situation, the heat source is the load that moves along the upper surface of the base sample. Therefore, we need to write the mobile heat source subroutine Dflux, and then add the Dflux subroutine to the job module to submit the analysis to realize the simulated laser quenching process.

\subsection{Simulation result analysis and test verification}

Abaqus saves the odb job after the analysis step is completed, and can observe the moving heat source moving the heating process and the temperature field cloud map on the model. In this paper, the cloud map (in ${ }^{\circ} \mathrm{C}$ ) of the temperature field at $0.1 \mathrm{~s}, 1.0 \mathrm{~s}$ and $1.76 \mathrm{~s}$ is intercepted, as shown in Fig. 4 below. It can be known from the temperature field distribution that during the laser quenching process, the temperature at the beginning of the laser quenching scanning path is significantly lower than the end of the laser quenching scanning path. The reason is the accumulation of heat flow, where the temperature is highest at the end of the spot. Analysis of the movement process and temperature field distribution of the spot shows that the temperature gradient of the front of the spot is significantly larger than the temperature gradient of the trailing edge of the spot along the direction of the laser scanning path. The reason is that during the laser scanning process, the back end of the heated end, that is, the trailing edge temperature of the spot is higher than the front end of the spot where the spot is not scanned, and the temperature difference from the center of the spot is inconsistent, and the rear edge of the heated spot is slower than the front of the spot. The temperature gradient is less than the temperature gradient of the front of the spot. The temperature of the center of the moving spot is above the quenching critical temperature, and the heating zone is instantaneously cold, so that the Austenite transforms to Martensite and achieves the purpose of laser quenching. Observing the loading of the moving heat source, the shape of the heat source spot is oblate, but the shape of the heat source spot defined in Fortran language compilation should be rectangular. The reason should be that there is residual and extension of heat flow before and after the heat source during heat source loading.

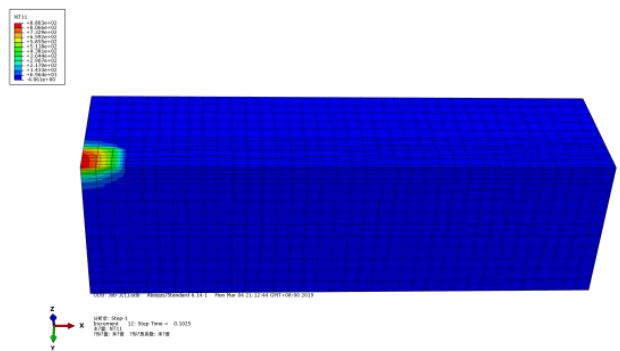

$\mathrm{T}=0.1 \mathrm{~s}$

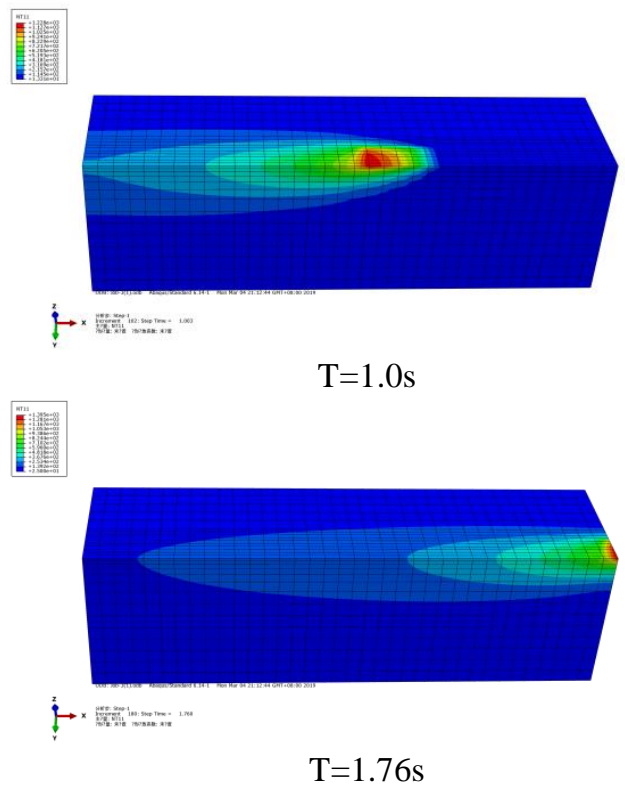

Fig.4. Heat source moving part of the time temperature field cloud map

When the intermediate time $\mathrm{t}=1 \mathrm{~s}$ is plotted, the temperature distribution curve of each node is perpendicular to the direction of the spot scanning path. The temperature field distribution is analyzed by the graph and the depth of the laser quench hardened layer is estimated by referring to the quenching critical point temperature.

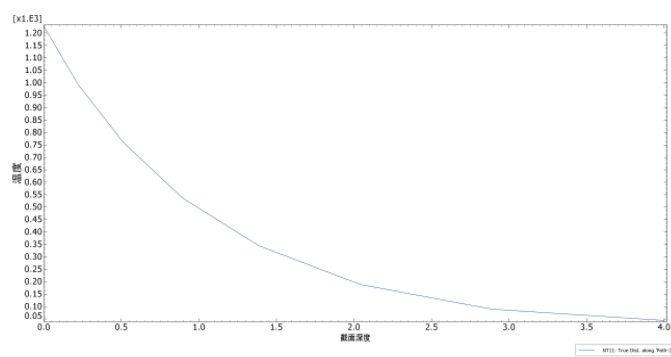

Fig.5. Depth temperature distribution curve

It can be seen from the depth temperature distribution curve that the depth section is the quenching critical point temperature at the node temperature of 850 . Therefore, the simulated laser quench hardening layer has a depth of about $0.45 \mathrm{~mm}$.

The test results are basically consistent with the simulation results, but the test results are slightly smaller than the simulation results. The reason for the low test result may be that the improper selection of the absorption rate of the fiber laser results in the input of heat. On the other hand, the physical property parameters of the materials calculated by means of software are not completely consistent with the actual ones.

\section{CONCLUSION}

In this paper, the Abaqus finite element software is used to simulate the laser quenching surface processing of high-phosphorus cast iron samples. The simulation results are basically consistent with the experimental results. Through the analysis of the simulation results, the temperature at the 
initial end of the laser scanning is significantly smaller than the temperature at the end of the laser scanning during the laser quenching process. The spot scanning speed is a crucial factor. It is necessary to accumulate heat at a low speed in the initial stage of scanning to ensure the depth of the hardened layer. At the end of the scanning, it is necessary to quickly avoid the influence of the heat flow accumulation effect to cause the temperature to be too high to melt the substrate.

In order to ensure the best laser quenching temperature of the matrix sample, it is necessary to change the laser process parameters. The DFlux mobile heat source subroutine written in Fortran can easily change the laser process parameters to simulate the quenching process, and realize the optimal process parameters for laser quenching. In this way, Abaqus is used to further simulate and study laser quenching, which provides a meaningful reference for experiments and processing.

\section{REFERENCES}

[1] Xu Hongwei,Pan Zhengxiang,Wen Degang.Research Status and Development of Laser Quenching Technology[J].China Printing \& Packaging Research,2014,6(02):1-8.

[2]Mdaniels R L, White S A, Liaw K, et al. Effects of a laser surface processing induced heat-affected zones on the fatigue behavior of AISI 4343 steel[J]. Materials Science and Engineering, 2008,485(25): 500-507.

[3]Stanislav. Microstructure and properties of cast iron after laser surface hardening[J]. Materiálové Inžinierstvo,2013,20(4): 153-159

[4]SHAN Jun,LI Min-juan,XU Hong-wei.Research on laser quenching control algorithm based on fuzzy control[J].Applied Laser,2015,35(01):40-43. [5] Huang Liangliang, Chen Liang, Tang Mingzhong, et al. Laser surface hardening and its numerical simulation research [J]. Heat Treatment Technology and Equipment, 2014, 35(1): 8-11.

[6] Wang Zhou, Jiang Chuanhai, Volker Schulze, Chen Yanhua, Qu Ning.Finite Element Analysis of Laser Quenching under Different Beam Moving Speeds[J].Materials Science and Technology,2011,19(04):95-102. [7]Hamar, R.,Kotlan, V.,Pánek, D.,Doležel, I.. 3D model of laser hardening of steel bodies with induction pre-heating[P]. Computation in Electromagnetics (CEM 2014), 9th IET International Conference on, 2014.

[8] Duradundi S B, Krishna S P. Application of the central composite design in optimization of laser transformation hardening parameters of commercially pure titanium using $\mathrm{Nd}$ :YAG laser[J]. International Journal of Advanced Manufacturing Technology, 2012, 59: 169-192.

[9] Yan Binggong, Liu Jichang, Guo Yihui. Analytical calculation of laser surface hardening depth of nodular cast iron[J].Laser Technology,2011,35(02):206-209.

[10] Huang Jian. Simulation of laser metal surface quenching thermal field [D]. Huazhong University of Science and Technology, 2007.

Shengwen Zhang is a professor and master advisor of Jiangsu University of Science and Technology. He received his Ph.D. degree in mechanical manufacturing in Jiangsu University in 2013. The main research directions are CAD/CAPP/CAM integration technology and Group Technology.

Zifan Xia is a Master student of Jiangsu University of Science and Technology. The main research directions was Laser surface strengthening technology 